Cinémas

Revue d'études cinématographiques

Journal of Film Studies

\title{
Le territoire de la critique
}

\section{Denis Bellemare}

Volume 6, numéro 2-3, printemps 1996

\section{La Critique cinématographique}

URI : https://id.erudit.org/iderudit/1000974ar

DOI : https://doi.org/10.7202/1000974ar

Aller au sommaire du numéro

Éditeur(s)

Cinémas

\section{ISSN}

1181-6945 (imprimé)

1705-6500 (numérique)

Découvrir la revue

Citer cet article

Bellemare, D. (1996). Le territoire de la critique. Cinémas, 6(2-3), 101-112. https://doi.org/10.7202/1000974ar

\section{Résumé de l'article}

La critique, c'est aussi de la théorie. Le dualisme que la pratique impose à la critique et à la théorie ne doit pas leur faire oublier leurs exigences communes de connaissance et de jugement. Dans un tel contexte d'écriture, quelles figures la critique du cinéma québécois tente-t-elle d'imprimer au territoire filmique? L'auteur cherche à bien définir une posture de critique d'imitation et d'identification. La critique et le film ne trouvent pas la distance nécessaire à leur propre symbolisation. Toutefois, la fin des années 80 laisse poindre un imaginaire nouveau du cinéma québécois obligeant lentement la critique à se repositionner. d'utilisation que vous pouvez consulter en ligne.

https://apropos.erudit.org/fr/usagers/politique-dutilisation/ 


\title{
Le territoire de la critique
}

\section{Denis Bellemare}

\begin{abstract}
RÉSUMÉ
La critique, c'est aussi de la théorie. Le dualisme que la pratique impose à la critique et à la théorie ne doit pas leur faire oublier leurs exigences communes de connaissance et de jugement. Dans un tel contexte d'écriture, quelles figures la critique du cinéma québécois tentet-elle d'imprimer au territoire filmique? Lauteur cherche à bien définir une posture de critique d'imitation et d'identification. La critique et le film ne trouvent pas la distance nécessaire à leur propre symbolisation. Toutefois, la fin des années 80 laisse poindre un imaginaire nouveau du cinéma québécois obligeant lentement la critique à se repositionner.
\end{abstract}

\section{ABSTRACT}

Criticism is also theory. The dualism that practice imposes on critics and theory should not obscure the common requirements of knowledge and judgment. In this context of writing, what figures does Québécois cinema criticism seek to imprint on this ground? The author addresses this issue through a discussion of the criticism of imitation and identification. Criticism and film don't manage the necessary distance for its own symbolization. Nevertheless the end of the 1980s brought a new imaginary to Québécois cinema which has slowly forced critics to reposition themselves.

Dans un récent numéro de la revue Spirale, Monica Haïm, présidente de l'Association québécoise des critiques de cinéma, a invité quelques théoriciens du nombre desquels je faisais partie et quelques critiques à livrer de part et d'autre ce qu'ils pensaient de la critique et de la théorie. L'échange a bel et bien eu lieu et, 
de la somme de ces écrits suite à une première exploration curieuse et collective, il s'est dégagé des séries de différences et de ressemblances selon des critères multiples et personnels, avec l'ambivalence propre aux gens du métier partageant un même territoire d'analyse. Mais cette ambiguïté et peut-être même cette duplicité n'origineraient-elles pas de la nature même d'un projet tentant de démarquer à tout prix critique et théorie?

Première observation après coup: la catégorisation des lieux des pratiques et de leurs institutions n'est que le phénomène ultime de leur parution et cette description analytique tout importante qu'elle soit ne doit pas préjuger d'une définition première plus essentielle et couvrir, à elle seule, tout ce champ définitoire. Ainsi, pour baliser des frontières, la réalité des modes de production ne peut pas se confondre avec le réel de la production critique et théorique comme mode d'écriture, ces deux instances étant de deux ordres parfois divergents. Les différences et les lois du genre de l'article critique et de l'essai théorique légitimés par les lieux d'une pratique institutionnalisée s'amenuisent, voire s'estompent, quand " [...] la question est celle des rapports entre l'activité critique et la littérature [ou le cinéma], ou si l'on veut, de savoir si le critique est ou n'est pas un écrivain" (Genette, p. 201).

Deuxième observation après coup: les études cinématographiques gagnent-elles à démarquer ces deux pratiques dans un même territoire alors que les critiques d'art tels Goodman, Greenberg, Payant et les critiques littéraires tels Thibaudet, Doubrovsky, Todorov et Northrop Frye sont aussi connus et perçus comme théoriciens? C'est dire que la critique, c'est de la théorie, et qu'elle ne peut être confondue à cause de ses hautes exigences d'éducation et de tradition culturelle à des comptes rendus périodiques ou des actualités journalières, à moins que ces chroniques exemplaires se donnent la hauteur de ces objectifs.

Car que penser d'une critique sans voix théorique, d'une théorie sans regard critique? Les paradigmes de la critique et de la théorie se regardent en chiens de faïence. Le plan d'attaque chez l'un correspond à une stratégie d'approche chez l'autre. Les formules incisives et éclairs de la critique se démarquent d'une rhétorique laborieuse et longue de la théorie. Les clichés de l'un 
renvoient au jargon de l'autre. La preuve, idée forte de la critique, est prise à parti par l'épreuve d'une méthodologie, véritable forteresse de la théorie. Et ainsi, le fondement d'un regard prégnant critique et théorique s'est évanoui dans la polarisation irrémédiable des arcanes du pouvoir, le théoricien critique doit faire son choix: ou les industries de la communication ou les institutions du savoir, ou l'association des critiques de cinéma ou l'association des études cinématographiques, ou les journaux et les revues de vulgarisation ou les revues spécialisées avec comité de lecture. Quand la forme courte de la critique judicieuse et la forme longue de l'article savant trouvent leur expression écrite en des institutions aussi socialement établies, cette formation symbolique comme loi du genre va au-delà de la pratique des individus où désormais il ne faut pas confondre le service à la collectivité avec une véritable activité de chercheurs. Mais cette unité duelle mine toutefois la théorie et la critique d'un même défaut: l'illusion du goût et/ou du savoir les prive et les éloigne d'une pensée profonde, vive et personnelle. Il faut bien saisir que la théorie et la critique sont des activités à deux versants, connaissance et jugement: dès lors, l'articulation de ces qualités devient par le retour même de l'écriture l'articulation nécessaire de la théorie et de la critique.

Mais cette polémique dynamique date en littérature depuis au moins la deuxième moitié du XIX ${ }^{e}$ siècle sans avoir renié à la critique son aspect théorique ni à la théorie son aspect critique. Déjà en 1930, Albert Thibaudet dans Physiologie de la critique parle d'une sorte de cohabitation où la pluralité de parties engagées et d'individus spécialisés forment un archipel de points de vue. Son essai faillit d'ailleurs s'intituler Géographie de la critique afin de bien rendre compte, écrit-il, de la critique des honnêtes gens, la critique des professionnels et la critique des artistes. Et avec un humour bien particulier, il sait bien l'art d'intégrer cette tension duelle de la critique entre professeurs "porte-toge» et journalistes "porte-plume».

Comme eau-mère de la critique, il ne faut pas compter seulement la formation d'une corporation de professeurs, la naissance d'une corporation de journalistes, mais aussi leur rivalité et leur opposition. Voilà un 
siècle qu'il existe une critique des professeurs et une critique des journalistes, sans que le sage doive s'en émouvoir plus que de voir coexister les brunes et les blondes, le bourgogne et le bordeaux (p. 9).

Ainsi pour Thibaudet, il s'agit réellement de la confection d'une chaîne où " [...] bon gré mal gré, la critique prend place à son rang, à son moment et dans son lieu, parmi une série d'autres ouvrages" (Poulet, p.10). Les critiques littéraire et artistique ont souvent animé et ont été au centre des grands mouvements sur les fondements théoriques de leur art. Les crises et les débats autour de la Nouvelle Critique en littérature dans les années 60 ont emporté avec elle ces "[...] ramassis de platitudes, d'incohérences et de clichés dont sont faits le bon goût et le beau style des commentaires traditionnels" (Doubrovsky, p. 242). Il ne s'agit plus de traduire l'original dans le banal, la critique n'est plus un appareil de contrôle, la critique s'assume comme écriture d'engagement et de connaissance. Pour Doubrovsky, la critique se définit un cadre de pensée plus qu'un système et la prétendue objectivité, le vraisemblable critique laissent place à la subjectivité transformée par sa propre pratique d'écriture: "Le moi travaillé du critique, comme celui de l'écrivain, n'est pas un ego replié et refermé sur un sentir individuel: en lui, le moi de nature a fait place au moi de culture" (p. 249). Dans Critique de la critique, Todorov avance l'idée de "critique dialogique" où le critique parle non seulement des œuvres mais aussi aux œuvres, ou plutôt avec les œuvres (p. 185).

Ce court passage par la littérature veut montrer les exigences et la perspective critique qu'une discipline peut se donner. Ainsi définir la critique uniquement par la pratique du milieu revient par tautologie à décrire le milieu et ses lois plutôt que les exigences d'une tradition culturelle de la critique. Mais en cela, il n'y a aucun mépris pour la réalité qui, elle, se débat avec l'air du temps. "La critique du jour, qui suit les écrivains [les cinéastes] du jour, et qui parle d'eux, et qui en parle du point de vue du jour, dans le langage du jour, contribue donc à donner de l'être au jour qui passe" (Thibaudet, p. 37).

La critique cinématographique offre un défilement spatiotemporel sur fond d'engagement géographique et historique. En 
un sens, l'on peut dire qu'elle explore beaucoup plus un territoire qu'elle ne l'occupe, ceci dit en d'autres mots, l'occupation de la critique vise justement à tracer ledit territoire en des points historiques et locaux bien marqués, à la manière des repérages des arpenteurs.

Ces tracés tout en pointillés que forme la critique se constituent en un véritable réseau de pôles énonciateurs, variables et dans l'espace et dans le temps. Les premiers critiques, à la manière des pionniers de la manivelle, se sont évertués à louer selon leurs propres idéologies et intérêts les mérites du cinématographe. Le journal catholique français de la fin du XIX siècle, Le Fascinateur, n'a-t-il pas poussé la métaphore religieuse du cinématographe comme mystère d'une résurrection? Ainsi de nombreux premiers écrits sur les toutes premières vues cinématographiques traduisent l'enthousiasme d'une perception exacerbée. Ricciotto Canudo, dans un autre registre, n'a-t-il pas voulu introduire cette expression d'une nouvelle technique dans le paradigme de l'art au-delà de la question du divertissement ? Antoine de Baecque, dans un article tout récent des Cabiers $d u$ cinéma, lui confere le titre de pionnier de la critique, de la théorie et de la cinéphilie: "[...] triple regard porté sur les images qui dessine dès l'origine les trois modes de l'attitude et de l'écritures cinéphiles: la véhémence dans le combat, l'ambition et la minutie du classement des œuvres, et le culte du jugement de goût paradoxal " (p. 52). Canudo, Delluc, Epstein ont en quelque sorte formulé cette question première: qu'est-ce que le cinéma? De ces multiples appropriations et approximations sont nées par accumulation, pour ne pas dire par sédimentation, les assises théoriques d'un Balazs consacrant le film comme œuvre et répondant à une autre question de la nature et de l'évolution de cet art nouveau : que peut le cinéma?

À partir de là, la critique peut pousser la question d'une machine désirante: que veut le cinéma? Et en ce sens, elle inscrit bien les sauts et les humeurs du temps comme un sismographe, à la manière parfois fulgurante d'écrits corsaires pour reprendre l'expression de Pasolini.

Tout comme le temps n'est pas une chose continue et tout comme les lieux s'ouvrent à de nombreux paysages, cimes et 
gouffres, l'expression nationale de la critique se trouve tout aussi éclatée. La question de la survie d'un cinéma au-delà de strictes questions de goût et de langage cinématographique peut se révéler un véritable débat identitaire. Le cinéma et la critique se trouvent alors au cœur de l'institution et de la nation.

\section{Une critique d'imitation et d'identification}

Quelles figures la critique d'ici imprime-t-elle, prise par les exigences d'une écriture liée à la connaissance et au jugement de l'histoire générale du cinéma et de l'histoire singulière du cinéma québécois?

Dans un résumé paru dans le Dictionnaire du cinéma québécois, Réal La Rochelle situe l'apparition des premières revues de cinéma après la Deuxième Guerre mondiale, au détour des années 50 , lors de la première vague commerciale du cinéma canadien-français (p. 415-420). Ces revues Découpages (19501955), Séquences (1955-...) visent «[...] une sorte d'appropriation communautaire" (Pagé, p. 46) du cinéma comme formation humaniste et chrétienne, ces approches prennent en compte la culture cinématographique en autant que le film soit "[...] esthétiquement remarquable et moralement beau" (Pagé, p. 69), et ce selon les critères religieux d'alors. Seule la revue Images (1955-1956) tente une réflexion plus ouverte à une culture cinématographique populaire et ainsi plus dégagée de l'idéologie catholique et de résistance de l'époque. Le monde de la critique cinématographique des années 50 se fond aux intentions de l'époque qui ne se pose pas encore la question nationale de son cinéma, et qui ne se pose la question du cinéma en général que par une sorte de travestissement religieux de valeurs humaines. Roger Caillois, dans Méduse et Cie, dépeint cette tendance à l'imitation comme " [...] assimilation au décor, au milieu, recherche de l'invisibilité. Pour parvenir à cette fin [de ne pas être remarqué], l'animal doit essentiellement perdre son individualité, c'est-à-dire effacer ses contours, les appareiller à un fond" (p. 102).

L'ouverture au cinéma pour mieux se l'approprier socialement dans le champ des pratiques culturelles et institutionnelles, c'est une chose de sociologie avec ses stratégies de production et de 
diffusion de la culture cinématographique. L'ouverture aux films pour mieux se les approprier cinématographiquement dans le champ des pratiques signifiantes, c'est une autre chose, de critique et de théorie. La première tient d'une posture socio-existentielle par rapport au cinéma, et les recherches de Benoît Pagé sur les revues de cinéma de cette période relèvent et révèlent les constructions sociales d'une telle appropriation. La seconde affiche une position cinématographique fondamentale non comblée par ces revues. Avec la revue Objectif(1960-1967) s'amorce un véritable virage cinéphilique à la mesure de la Nouvelle Vague du cinéma français, de l'émergence du cinéma québécois en plein cœur de la Révolution tranquille et d'où surgit «[...] la clarification et la formation d'une pensée cinématographique " (Lever, p. 80). La revue Séquences se rallie aussi à sa manière à ce nouveau tournant, le cinéaste Jean-Pierre Lefebvre, alors collaborateur à la revue, lie le cinéma à la culture nationale: "Le temps de vivre est venu, celui de nous former une conscience et une culture enracinée: le temps de combattre pour nos idées, pour nos goûts, pour les arts et plus particulièrement pour le cinéma" (p. 26). Mais de tous ces écrits n'émerge pas un continent théorique autonome. "À juste titre, Yves Lever analyse l'influence de la critique française sur les revues québécoises comme suit: Henri Agel et Amédée Ayfre sur Séquences; Positif et les Cabiers du cinéma (première manière) sur Objectif; Cinéthique et les Cahiers du cinéma (deuxième manière) sur Champ Libre» (La Rochelle, 1988, p. 418).

Lors des années 70 et début 80 , ces stratégies d'appropriation, ces influences extérieures vont pousser leurs ramifications mimétiques de façon plus complexe à l'intérieur de ce que Todorov nommerait une critique d'identification tentant de " [...] se projeter dans l'autre au point d'être en état de parler en son nom " (p. 185). La critique tout comme le film ne trouvent plus la distance nécessaire à leur propre symbolisation, à leur propre signification, la critique tout comme le film suivent à la lettre leur enregistrement sans levée de sens autre que le signifié lui-même. La critique reprend et répète le film qui reprend et répète la réalité. Ainsi, certaines analyses socio-historiques, par exemple, confondent souvent l'image du corps social dans sa présentation 
au monde au corps même de l'image visuelle dans le monde de la représentaton filmique. Nous sommes loin de penser qu'il y a symétrie entre ces deux mondes et qu'au contraire, une certaine asymétrie aiderait à les symboliser; le travail de la critique tout comme le travail du film consisteraient en une véritable mise en œuvre du signifiant plutôt qu'à une imitation appliquée du référent au signifié. Il y a ainsi dans le cinéma québécois et dans la critique des années 70 et du début des années 80 un certain parallélisme d'imitation des formes de contenu et des formes d'expression transparaissant dès lors comme la pire des résistances au langage. Le réalisme du cinéma québécois ainsi qu'une majorité de ses écrits analytiques masquent, camouflent, par une sorte d'imitation défensive, une forme symbolique pouvant leur révéler une continuité profonde, méconnue, refoulée. À venir jusqu'ici, l'histoire du cinéma québécois s'est révélée en grandes tranches discontinues juxtaposées à ses grandes périodes sociopolitiques, et de même "[...] symptomatiquement, chaque décennie a ses revues" (La Rochelle, 1988, p. 415). La revue Cinéma Québec (1971-1978) s'avéra le château fort de cette critique d'identification redoublée d'une critique identitaire d'un cinéma national et elle constitue un passage obligé pour une étude parallèle d'un cinéma, de sa société, de sa critique comme stade du miroir comme formation du Je. La revue Champ Libre (1971-1973) se veut l'envers de cette critique d'identification et croit à la " [...] prise en charge du cinéma, du film comme outil de conscientisation, de son rôle idéologique instrumental " ( $\mathrm{La}$ Rochelle, 1994, p. 102). Un peu plus tard, la revue anglophone Ciné-Tracts (1977-1982), forte de la présence de Ron Burnett et de ses échanges avec des théoriciens britanniques et les féministes américaines, annonce les grands mouvements théoriques, et encore influents, des années 80 .

Imiter, pasticher, ce n'est certes pas encore critiquer, mais c'est déjà rassembler et recréer, deux actions qui constituent le premier temps de la pensée critique. Filmer, enregistrer, tout reproduire, ce n'est certes pas encore du cinéma, mais les cinéastes tels les chroniqueurs de la rhétorique ancienne, le scriptor, le compilator, le commentator, inventorient leur territoire, l'arpentent, le recensent pour se reconnaître, pour se 
connaître. Jamais la société québécoise n’a été aussi filmée, jamais cinéma n'a été aussi quantitativement mesuré sous forme de rapports statistiques, dictionnaires, annuaires, répertoires analytiques.

Le mouvement consiste à glisser le plus près possible de la surface du réel, de l'étaler dans son évidence même en atténuant l'espace entre sa reproduction et ses manifestations. Il s'agit d'arpenter le trajet du littéral, de prélever toutes les singularités qui, dans leurs additions, schématisent le contexte social. Le cinéma québécois dans sa persévérante et obsédante vitalité à recenser, à répertorier ses images rejoint quelque part cette poussée obsessive du banal vers le réel dans ce qu'il y a de descriptif, de reproduisible. À dresser ainsi une emprise du factuel et de l'actuel, ce désir de s'inscrire dans le réel pour le construire le dresse aussi contre d'autres voies.

Le fait qu'il n'existe à peu près pas de genres dans le cinéma québécois explique peut-être l'approche plutôt monologique à laquelle la critique l'a confiné. En effet, on a rarement tenu compte de ce cinéma dans une perspective autre que socio-historique. Le point de vue poétique, le point de vue qui suppose que le cinéma n'est pas qu'un miroir que l'on promène sur la société, n'existe à peu près pas dans les études sur le cinéma québécois. Cette manière de voir n'aurait rien de condamnable si elle avait aussi donné lieu, à côté de l'approche majoritairement référentialiste, à une approche poétique. [...] De plus, comme beaucoup de cinématographies tardives et minoritaires, il y a un critère suffisant à l'identification de cette cinématographie: celui de sa nationalité (Garneau, p. 94).

L'histoire critique et théorique du cinéma québécois a souvent mis à l'écart, voire pris à parti la part imaginaire de son cinéma comme si l'esthétique du réel n'en indiquait pas, par le revers contradictoire de sa position, une orientation encore innommée, peut-être inachevée, et somme toute particulière.

La question de l'imaginaire dans le cinéma québécois resurgit ces dernières années dans de nombreux écrits. Elle ne s'avance jamais bien simplement et facilement, elle s'annonce au pluriel dans "Imaginaires du cinéma québécois" de la Revue belge $d u$ 


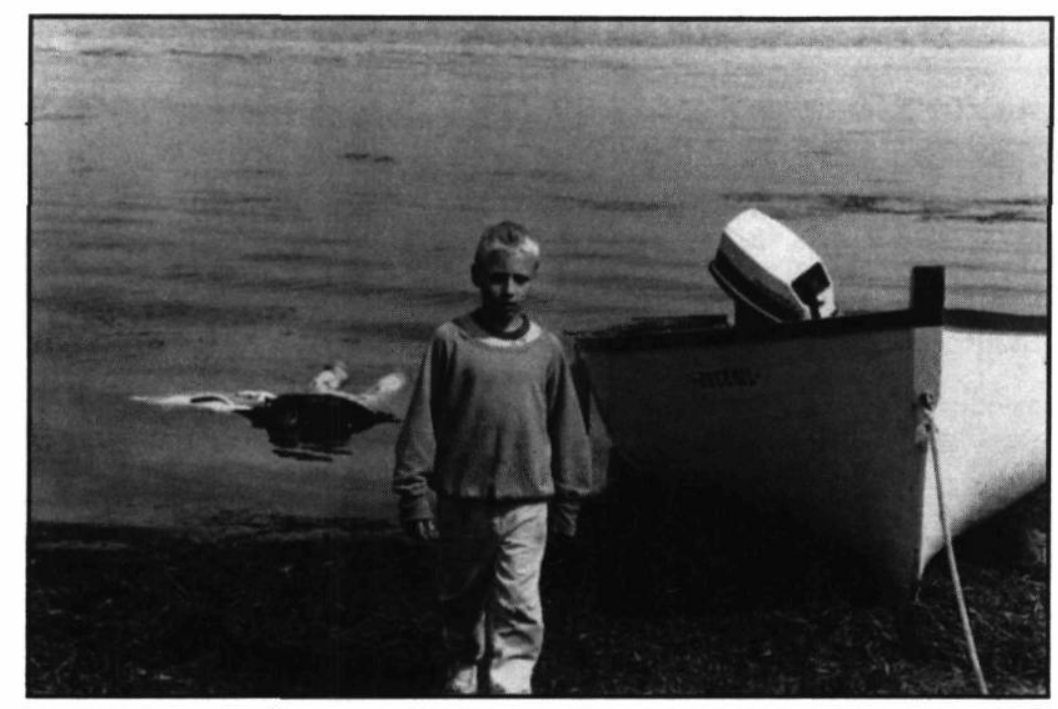

\section{Cargo de François Girard (1990)}

Collection Clinémathèque québécoise

cinéma $\left(\mathrm{n}^{\circ} 27\right.$, automne 1989), elle se pose en interrogation dans l'" Imaginaire québécois?" de la revue Lumières $\left(\mathrm{n}^{\circ} 27\right.$, été 1991), elle s'énonce dans Cinéma de l'imaginaire québécois de Heinz Weinmann autour de la problématique du "roman familial» de Freud. Il y a un nouveau cinéma pour le dire: JeanClaude Lauzon, François Girard, Léa Pool, Jeanne Crépeau et combien d'autres que les revues 24 Images (1979-...) et CinéBulles (1980-...) suivent et nous font connaître dans leurs multiples dossiers. Il ne manque plus qu'une Nouvelle Critique du cinéma québécois.

\section{Le passeur}

Si la critique constitue une discipline bien particulière, le critique représente un personnage bien singulier. "Ce qu'on peut déplorer, c'est le refus du critique de se poser soi-même en sujet réfléchissant (plutôt que de s'effacer derrière l'accumulation de faits objectifs) et de porter des jugements" (Todorov, p. 186). "Rêver d'être une glace sans tain, un miroir ou même une loupe, c'est pour le critique une dérobade, qui détruit le sens même de la communication" (Doubrovsky, p. 246). 
S'il y eut un critique qui parla aux œuvres, des œuvres, avec les œuvres, ce fut bien Patrick Straram dit le Bison ravi (19341988). Un vivre selon sa critique, aurait-il écrit. Il fut exemplaire de sa subjectivité même travaillant l'œuvre et autant travaillée par elle. Le désir critique de Patrick Straram est un désir de cinéma et un désir du monde, souhaitant comme Rivette et comme Claude-Jean Philippe "[...] que le monde réel offert sur l'écran soit aussi une idée du monde et que la présence humaine sur l'écran soit la présence humaine au monde" (Straram, 1971a, p. 7). Car sans critique et sans discours, toutes les censures et tous les pouvoirs décident. Il avait sa famille nombreuse de cinéastes, de musiciens, de poètes, de philosophes d'où les innombrables citations: "Inutile de questionner mon sens critique s'il n'y a pas présence de ces présences [...]" (Straram, 1984, p. 17). Pour lui, le cinéma et la critique appartiennent à la même figure emblématique que fut Godard :

En tant que critique, je [Jean-Luc Godard] me considérais déjà comme cinéaste. Aujourd'hui, je me considère toujours comme critique, et, en un sens, je le suis plus encore qu'avant (Straram, 1971a, p.12).

Straram est le premier critique qui ait eu ici une conscience tricontinentale. Et une conscience tout court. Qu'il soit ici salué (Noguez, p. 137).

\section{Université du Québec à Chicoutimi}

\section{OUVRAGES CITÉS}

Baecque, Antoine de. "Canudo, premier cinématophile». Cahiers du cinéma, n" 498 (1996).

Caillois, Roger. Méduse et Cie. Paris: Gallimard, 1979.

Doubrosky, Serge. Pourquoi la nouvelle critique? Critique et objectivité. Paris: Mercure de France, 1966.

Garneau, Michèle. "Minorité et généricité». Iris, n 20 (1995) p. 93-103.

Genette, Gérard. "Raisons de la critique pure", dans Georges Poulet (direction), Les Chemins actuels de la critique. Paris: Union générale d'éditions, 1968.

La Rochelle, Réal. "Revues de cinéma", dans M. Jean et M. Coulombe (direction), Le Dictionnaire du cinéma québécois. Montréal : Boréal (1988) p. 415-420.

La Rochelle, Réal. Cinéma en rouge et noir. Montréal: Triptyque, 1994.

Lefebvre, Jean-Pierre. "Les jeunes et le cinéma canadien". Séquences, n 29 (1962) p. 26-27. 
Lever, Yves. "La revue Objectif(1960-1967)", Dialogue, cinéma canadien et cinéma québécois. Montréal: Médiatexte Publications Inc. / La Cinémathèque québécoise (1987) p. 73-81.

Noguez, Dominique. "Cinémarx \& Rolling Stone". Champ libre, n 1 (1971).

Pagé, Benoît. Critiques cinématographiques et institutions du cinéma québécois (19501962). Québec: Laboratoire de recherches sociologiques/ Université Laval, 1993.

Poulet, Georges (direction). Les Chemins actuels de la critique. Paris: Union générale d'éditions, 1968.

Straram, Patrick. ONE + ONE Cinémarx \& Rolling Stone. Montréal: Les Herbes rouges, 1971a.

Straram, Patrick et Jean-Marc Piotte. Gilles cinéma Groulx le lynx inquiet. Montréal: Cinémathèque québécoise / Éditions québécoises, $1971 \mathrm{~b}$.

Straram, Patrick. "Blues clair/ Bande à part, Québec", dans Michel Larouche (direction), "Cinéma québécois. Nouveaux courants, nouvelles critiques". Dérives, n 52 (1986) p. 67-83.

Straram, Patrick. "Blues clair Salut, l'anar". Les dossiers de la cinémathèque, n 12 (1984) p. 17-20.

Thibaudet, Albert. Physiologie de la critique. Paris: La Nouvelle Revue critique, 1930.

Todorov, Tzvetan. Critique de la critique, un roman d'apprentissage. Paris: Seuil, 1984.

Weinmann, Heinz. Cinéma de l'imaginaire québécois. Montréal : l'Hexagone, 1990. 